\title{
On Normality of a Family of Holomorphic Functions
}

By

\section{Chikara WATANABE*}

0. On normality of a family of continuous functions, the theorem of Ascoli-Arzelà is well known. Especially, if we include the case of compact divergence, for a family of holomorphic functions, normality follows from the condition of equicontinuity at each point. In this paper we consider the normality in the wide sense. For a compact metric space $K$, we can construct another compact metric space $\ll \operatorname{Comp}(K) \gg$ consisting of all closed subsets of $K$. Using this space, in a $\sigma$-compact, locally compact metric space $\mathrm{X}$, we can define the concept of convergence of a sequence of closed subsets of $\mathrm{X}$ and by means of this convergence, every family of closed subsets is always normal. As a consequence of this fact, we can prove that every family of continuous mappings from a connected, $\sigma$-compact and locally compact metric space to another $\sigma$-compact, locally compact metric space with some additional condition is normal if it is equicontinuous at each point. The method of our proof described in the section 1 seems to be interesting.

On normality of a family of holomorphic functions, many results are obtained by G. Julia [6]. From them, we denote the following interesting theorem:

Let $\mathfrak{F}$ be a family of holomorphic functions in a domain in $\boldsymbol{C}^{2}$. If every function $f \in \mathfrak{F}$ does not take two fixed different values, then $\mathfrak{F}$ is normal.

Now, if we consider the normality of a family of holomorphic functions in a domain $D$ in the strict sense, that is, if every sequence of the family

Communicated by S. Hitotumatu, June 11, 1973. Revised July 16, 1973.

* Department of Mathematics, College of Liberal Arts, Kanazawa University, Kanazawa. 
has a subsequence which converges uniformly to a holomorphic function on every compact set in $\mathrm{D}$, then the domain of normality is a domain of holomorphy. This is known as «Conjecture of Julia». Using the word graph of holomorphic function, this is described in other words as follows.

Let $\mathfrak{F}$ be a family of holomorphic functions in a domain D in $\mathbb{C}^{n}$. Suppose that the set $\mathfrak{F}(p)=\{f(p) ; f \in \mathfrak{F}\}$ is bounded for every point $p \in \mathrm{D}$. If $\mathrm{D} \times \boldsymbol{C}$ is a domain of normality of the family $\mathbb{S}_{\mathfrak{F}}$ of graphs of $\mathfrak{F}$, then $\mathrm{D}$ is a domain of holomorphy.

We shall give a proof of the above in the section 2 .

In the section 3 , we give an application of the theorem of Julia cited above. If $\mathrm{D}$ is a domain in the complex plane, then we get the converse of the theorem of Hurwitz.

1. In this section we consider a $\sigma$-compact, locally compact metric space $\mathrm{X}=\left(\mathrm{X}, d_{\mathrm{X}}\right)$, where $d_{\mathrm{X}}$ is a metric in $X$. Let $\left\{E_{j}\right\}$ be a sequence of non empty closed subsets of $\mathrm{X}$.

Definition 1. The sequence $\left\{E_{j}\right\}$ converges geometrically to a closed set $E$ in $\mathrm{X}$ if and only if:

(i) If $E$ is not empty then for any point $p \in E$, there exists a sequence $\left\{p_{j}\right\}$ such that $p_{j} \in E_{j}$ and $p_{j} \rightarrow p$.

(ii) For any compact set $K$ in $\mathrm{X}$ with $K \cap E=\phi$, there exists a $j$ 。 such that $K \cap E_{j}=\phi$ for all $j \geqq j_{\circ}$.

From this definition we have immediately:

(1) Any subsequence of $\left\{E_{j}\right\}$ also converges geometrically to $E$.

(2) If a sequence $\left\{p_{j}\right\}$ with $p_{j} \in E_{j}$ has accumulating points, then they belong to $E$.

(3) The condition (ii) is equivalent to the following:

(ii)' For any positive number $\varepsilon$ and for any compact set $K$ in $\mathrm{X}$, there exists $a j$ 。 such that for all $j \geqq j_{0}$, the set $E_{j} \cap K$ is included in the set $E^{(\varepsilon)} \cap K$, where $E^{(\varepsilon)}=\cup\left\{q \in \underset{p \in E}{\operatorname{X}} ; d_{\mathrm{X}}(q, p)<\varepsilon\right\}$.

In fact, (1) and (2) are direct consequences of the definition. We shall show (3). 
(ii) $\rightarrow(\text { ii })^{\prime}:$ We may assume that $E$ is not empty. Suppose that there exists a positive number $\delta$ and a compact set $K$ such that the set $\left\{j ; E_{j} \cap K\right.$ $\left.-E^{(\delta)} \neq \phi\right\}$ of positive integers is an infinite set. For simpliciy, we may assume that $E_{j} \cap K-E^{(\delta)} \neq \phi$ for all $j$. Let $\left\{p_{j}\right\}$ be a sequence such that $p_{j} \in E_{j} \cap K-E^{\delta}$. Since $K$ is compact, this sequence has accumulating points. Let $p$ be one of them. Then $p \in E$ by the property (2). Thus the set $B_{\mathrm{X}}(p: \delta)=\left\{q \in \mathrm{X} ; d_{\mathrm{X}}(q, p)<\delta\right\}$ is included in $E^{(\delta)}$ and $p_{j} \in B_{\mathrm{X}}(p: \delta)$ for all sufficiently large $j$. This is a contradiction.

(ii)' $\rightarrow$ (ii): Let $K$ be a compact set with $K \cap E=\phi$. Then there exists a positive number $\varepsilon$ such that $K \cap E^{(\varepsilon)}=\phi$. Thus there exists a $j$ 。 such that $E_{j} \cap K=\phi$ for all $j \geqq j_{\circ}$.

Here we explane the necessary fundamentals of the notion of the space Comp $(K)^{1)}$. Let $K$ be a compact metric space. For two non empty closed subsets $F_{1}, F_{2}$ of $K$, we define the metric $\widehat{d}$ between $F_{1}$ and $F_{2}$ by

$$
\breve{d}\left(F_{1}, F_{2}\right)=\sup _{x \in F_{1}} \inf _{y \in F_{2}} d(x, y)+\sup _{t \in F_{2}} \inf _{s \in F_{1}} d(s, t),
$$

where $d$ is a metric in $K$.

This metric is called Hausdorff metric. From this metric, Comp $(K)$, the set of all non empty closed subsets of $K$, becomes a compact metric space $^{2)}$. If $\left\{E_{j}\right\}$ converges to $E$ in Comp $(K)$, the following two properties are easily verified:

(i) For any point $p \in E$, there exists a sequence $\left\{p_{j}\right\}$ such that $p_{j} \in E_{j}$ and $p_{j} \rightarrow p$.

(ii) For any sequence $\left\{p_{j}\right\}$ with $p_{j} \in E_{j}$, the accumulating points of $\left\{p_{j}\right\}$ belong to $E$.

Now let $\left\{K_{j}\right\}$ be an exhaution of X by compact sets: $K_{1} \ll K_{2} \ldots, \cup K_{j}=$ X. Take a sequence $\left\{E_{j}\right\}$ of closed subsets of X. For any compact set $K_{\nu}$, if the set $\left\{j ; E_{j} \cap K_{\nu} \neq \phi\right\}$ of positive integers is finite, we define that $\left\{E_{j}\right\}$ converges to a null set. Otherwise, there exists a $j$ 。 such that $\left\{j ; E_{j} \cap K_{j}\right.$ 。 $\neq \phi\}$ is an infinite set. For simplicity, we may assume that $E_{j} \cap K_{j_{0}} \neq \phi$ for all $j$. From the compactness of the space Comp $\left(K_{j_{0}}\right)$, we can choose

1) More precisely see [1], [7].

2) See [1] section 5, Chapter 2. 
a subsequence $\left\{E_{j}^{\left(j_{0}\right)}\right\}$ of $\left\{E_{j}\right\}$ such that $\left\{E_{j}^{\left(j_{0}\right)} \cap K_{j_{0}}\right\}$ converges to a closed set $F_{j_{0}}$ in Comp $\left(K_{j_{0}}\right)$. From the sequence $\left\{E_{j}^{\left(j_{\circ}\right)}\right\}$ we can also choose a subsequence $\left\{E_{j}^{\left(j_{o}+1\right)}\right\}$ such that $\left\{E_{j}^{\left(j_{0}+1\right)} \cap K_{j_{0}+1}\right\}$ converges to a closed set $F_{j_{0}+1}$ in Comp $\left(K_{j_{0}+1}\right)$. We proceed this process. Then it is easily seen that

$$
E=\cup F_{j_{0}+j-1}
$$

is closed and that the diagonal sequence $\left\{E_{j}^{\left(j_{\circ}+j-1\right)}\right\}$ converges geometrically to $E$. Consequently we have

Lemma 1. Let $\mathrm{X}$ be a $\sigma$-compact, locally compact metric space. Then every family of closed subsets of $\mathrm{X}$ is normal in the sense of geometric convergence.

Now let $\mathrm{Y}=\left(\mathrm{Y}, d_{\mathrm{Y}}\right)$ be a $\sigma$-compact metric space which satisfies the following condition:

For any compact set $K \subset \mathrm{Y}$ and for any positive number $R$, the set

$$
\left.\overline{K^{(R)}}=\overline{\bigcup_{p \in K}\left\{q \in \mathrm{Y} ; d_{\mathrm{Y}}(q, p)<R\right.}\right\}
$$

is compact.

The space $\mathrm{Y}$ which satisfies the above condition is necessarily locally compact. Let $\mathfrak{F}$ be a family of continuous mappings from the connected, locally compact and $\sigma$-compact metric space $\mathrm{X}$ to $\mathrm{Y}$ cited above.

Definition 2. $\mathfrak{F}$ is called equicontinuous at $p \in \mathrm{X}$ if and only if given any positive number $\varepsilon$, there exists a positive number $r$ such that $d_{\mathrm{Y}}\left(f\left(p^{\prime}\right)\right.$, $f(p))<\varepsilon$ for all $f \in \mathfrak{F}$ and for all $p^{\prime} \in B_{\mathrm{X}}(p: r)$.

Let $C(\mathrm{X}, \mathrm{Y})$ be the set of all continuous mappings from $\mathrm{X}$ to $\mathrm{Y}$. The topology on $C(\mathrm{X}, \mathrm{Y})$ is the usual compact uniform topology.

Definition 3. A sequence $\left\{f_{j}\right\}$ of $C(\mathrm{X}, \mathrm{Y})$ is called to be compact divergence if and only if given any compact set $K$ in $\mathrm{X}$ and compact set

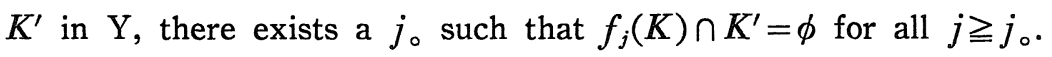

Definition 4. $\mathfrak{F}$ is called to be normal if and only if given a sequence 
of $\mathfrak{F}$, there exists a subsequence which is either compact divergence or compact uniform convergence.

Theorem 1. If $\mathfrak{F}$ is equicontinuous at each point of $\mathrm{X}$, then $\mathfrak{F}$ is normal.

Proof. Let $\mathbb{S}_{f}$ be a graph of $f \in \mathfrak{F}$, i.e., $\mathbb{S}_{f}=\{(p, f(p)) \in \mathrm{X} \times \mathrm{Y} ; p \in$ $\mathrm{X}\}$. Since $f$ is continuous $\mathscr{B S}_{f}$ is closed. Let $\mathbb{B}=\left\{\mathscr{B}_{f} ; f \in \mathfrak{F}\right\}$ be a family of graphs and let $d$ be the metric in $\mathrm{X} \times \mathrm{Y}$ defined by

$$
d\left((p, q),\left(p^{\prime}, q^{\prime}\right)\right)=\left\{d_{\mathrm{X}}\left(p, p^{\prime}\right)^{2}+d_{\mathrm{Y}}\left(q, q^{\prime}\right)^{2}\right\}^{1 / 2}
$$

then $\mathrm{X} \times \mathrm{Y}$ becomes a $\sigma$-compact, locally compact metric space. Thus by Lemma $1, \mathbb{S}$ is normal in $\mathrm{X} \times \mathrm{Y}$. Take a sequence $\left\{f_{j}\right\}$ from $\mathfrak{F}$. Since $\mathbb{B}$ is normal we can choose a sequence $\left\{\mathscr{S} \nu_{j}\right\}$ such that $\left\{\mathscr{S} \nu_{j}\right\}$ converges geometrically to a closed set $S$ in $\mathrm{X} \times \mathrm{Y}$, where $\mathscr{S} \nu_{j}=\mathscr{S} \dot{f}_{f_{j}}$. For convenience, we may assume that $\left\{\mathscr{\&}_{j}\right\}$ itself converges geometrically to $S$. For a point $p_{\circ} \in \mathrm{X}$, put

$$
S\left(p_{\circ}\right)=\left\{\left(p_{\circ}, q\right) \in \mathrm{X} \times \mathrm{Y} ; q \in \mathrm{Y}\right\} \cap S
$$

Then we shall show

Fact 1. $S\left(p_{\circ}\right)$ contains at most one point.

In fact, suppose that $\left(p_{\circ}, q\right),\left(p_{\circ}, q^{\prime}\right)$ be two different points of $S\left(p_{\circ}\right)$. Since $\left\{\mathcal{S}_{j}\right\}$ converges to $S$ geometrically, there exists two sequences $\left\{\left(p_{j}, q_{j}\right)\right\},\left\{\left(p_{j}^{\prime}, q_{j}^{\prime}\right)\right\}$ such that they converge to $\left(p_{\circ}, q\right)$ and $\left(p_{\circ}, q^{\prime}\right)$ respectively. From the equicontinuity of $\mathfrak{F}$ at $p_{0}$, there exists a positive number $r$ such that

$$
d_{Y}\left(f_{j}(p), f_{j}\left(p_{\circ}\right)\right)<\varepsilon=\frac{1}{8} d_{Y}\left(q, q^{\prime}\right)
$$

for all $p \in B_{\mathrm{X}}\left(p_{\circ}: r\right)$ and for all $j$. Since $p_{j} \rightarrow p_{\circ}, p_{j}^{\prime} \rightarrow p_{\circ}, q_{j} \rightarrow q$ and $q_{j}^{\prime} \rightarrow q^{\prime}$ respectively, there exists a $j_{\circ}$ such that $d_{\mathrm{X}}\left(p_{j}, p_{\circ}\right)<r, d_{\mathrm{X}}\left(p_{j}^{\prime}, p_{\circ}\right)$ $<r, d_{\mathrm{Y}}\left(q_{j}, q\right)<\varepsilon, d_{\mathrm{Y}}\left(q_{j}^{\prime}, q^{\prime}\right)<\varepsilon$ for all $j \geqq j_{\circ}$. Then it holds that

$$
d_{Y}\left(q, q^{\prime}\right) \leqq d_{Y}\left(q, q_{j}\right)+d_{Y}\left(q_{j}, f_{j}\left(p_{\circ}\right)\right)+d_{Y}\left(f_{j}\left(p_{0}\right), q_{j}^{\prime}\right)
$$




$$
+d_{Y}\left(q_{j}^{\prime}, q^{\prime}\right)<4 \varepsilon=\frac{1}{2} d_{Y}\left(q, q^{\prime}\right)
$$

Since $q \neq q^{\prime}$ this is a contradiction and then Fact 1 is proved.

Put $e=\{p \in \mathrm{X} ; S(p)=\phi\}$. Then

Fact 2. $e$ is open.

To prove this, we have only to show the following

Lemma 2. $p \in e$ if and only if the sequence $\left\{f_{j}(p)\right\}$ is a divergent sequence, i.e., for any compact set $K \subset \mathrm{Y}$, there exists $a j_{\circ}$ such that $f_{j}(p)$ $\notin K$ for all $j \geqq j_{\circ}$.

In fact, suppose that Lemma 2 is proved. Let $p_{\circ} \in e$. Take a positive number $r$ such that $d_{\mathrm{Y}}\left(f_{j}(p), f_{j}\left(p_{\circ}\right)\right)<1$ for all $p \in B_{\mathrm{X}}\left(p_{\circ}: r\right)$. Let $K$ be a compact set in $\mathrm{Y}$. Then there exists a $j_{\circ}$ such that $f_{j}\left(p_{\circ}\right) \notin \overline{K^{(2)}}$ for all $j \geqq j_{\circ}$. For every $p \in B_{\mathbf{X}}\left(p_{\circ}: r\right)$, since $K$ is compact there exists a $q_{j} \in K$ such that $d_{Y}\left(f_{j}(p), K\right)=d_{Y}\left(f_{j}(p), q_{j}\right)$, where of course $q_{j}$ depends on $p$. Then we have

$$
\begin{aligned}
d_{\mathrm{Y}}\left(f_{j}(p), K\right) & =d_{\mathrm{Y}}\left(f_{j}(p), q_{j}\right) \geqq d_{\mathrm{Y}}\left(q_{j}, f_{j}\left(p_{\circ}\right)\right)-d_{\mathrm{Y}}\left(f_{j}(p), f_{j}\left(p_{\circ}\right)\right) \\
& \geqq d_{\mathrm{Y}}\left(f_{j}\left(p_{\circ}\right), K\right)-1>1 .
\end{aligned}
$$

Therefore $f_{j}(p) \notin K$ for all $j \geqq j_{\circ}$. This means that $B_{\mathbf{X}}\left(p_{\circ}: r\right) \subset e$ and Fact 2 is proved.

Proof of Lemma 2. Let $p \in e$. If $\left\{f_{j}(p)\right\}$ is not a divergent sequence, there exists a subsequence $\left\{f_{\nu_{j}}(p)\right\}$ which converges to some point $q \in \mathrm{Y}$. Since $\left\{\left(p, f_{\nu_{j}}(p)\right)\right\}$ converges to $(p, q)$ and since $\left(p, f_{\nu_{j}}(p)\right) \in \mathbb{B S} \nu_{j}$, we have that $(p, q) \in S$. Thus $S(p) \neq \phi$ and $p \notin e$. This is a contradiction.

Conversely, let $\left\{f_{j}(p)\right\}$ be a divergent sequence. If $(p, q) \in S$, then there exists a sequence $\left\{\left(p_{j}, q_{j}\right)\right\}$ such that $\left(p_{j}, q_{j}\right) \in \mathcal{S}_{j}, p_{j} \rightarrow p$ and $q_{j} \rightarrow q$. Let $r$ be a positive number such that $d_{\mathrm{Y}}\left(f_{j}\left(p^{\prime}\right), f_{j}(p)\right)<1$ for all $p^{\prime} \in$

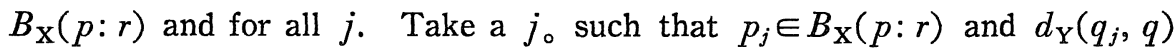
$<1$ for all $j \geqq j_{\circ}$. Then we have 


$$
\begin{aligned}
d_{\mathrm{Y}}\left(f_{j}(p), q\right) \leqq & d_{\mathrm{Y}}\left(f_{j}(p), q_{j}\right)+d_{\mathrm{Y}}\left(q_{j}, q\right)=d_{\mathrm{Y}}\left(f_{j}(p), f_{j}\left(p_{j}\right)\right) \\
& +d_{\mathrm{Y}}\left(q_{j}, q\right)<2
\end{aligned}
$$

for all $j \geqq j_{0}$. This implies that $\left\{f_{j}(p)\right\}$ is not a divergent sequence. This is a contradiction and then $S(p)=\phi$.

Fact 3. $\mathrm{X}-e$ is open.

In fact, let $p_{\circ} \in \mathrm{X}-e$, then $S\left(p_{\circ}\right)$ consists of exactly one point $\left(p_{\circ}, q_{\circ}\right)$. Take a sequence $\left\{\left(p_{j}, q_{j}\right)\right\}$ such that $\left(p_{j}, q_{j}\right) \in \mathbb{S}_{j}, p_{j} \rightarrow p_{\circ}$ and $q_{j} \rightarrow q_{\circ}$. Take a positive number $r$ such that $d_{Y}\left(f_{j}(p), f_{j}\left(p_{\circ}\right)\right)<\frac{1}{3}$ for all $p \in$ $B_{\mathrm{X}}\left(p_{\circ}: r\right)$ and for all $j$. There exists a $j_{\circ}$ such that $d_{\mathrm{Y}}\left(q_{j}, q_{\circ}\right)<\frac{1}{3}$, $d_{\mathrm{X}}\left(p_{j}, p_{\circ}\right)<r$ for all $j \geqq j_{\circ}$. Then

$$
d_{\mathrm{Y}}\left(f_{i}(p), q_{\circ}\right) \leqq d_{\mathrm{Y}}\left(f_{j}(p), f_{j}\left(p_{\circ}\right)\right)+d_{\mathrm{Y}}\left(f_{j}\left(p_{\circ}\right), q_{j}\right)+d_{\mathrm{Y}}\left(q_{j}, q_{\circ}\right) \leqq 1
$$

for all $p \in B_{\mathbf{X}}\left(p_{\circ}: r\right)$ and for all $j \geqq j_{\circ}$. This means that $\left\{f_{j}(p)\right\}$ contains a convergent sequence for all $p \in B_{\mathrm{X}}\left(p_{0}: r\right)$, so that $B_{\mathrm{X}}\left(p_{\circ}: r\right) \subset \mathrm{X}-e$. Thus $\mathrm{X}-e$ is open.

Since $\mathrm{X}$ is connected, as a result of these facts we have that $e=\phi$ or $e=\mathrm{X}$.

(1) In the case that $e=\mathrm{X}$ :

Let $K_{1}, K_{2}$ be two compact sets in $\mathrm{X}$ and $\mathrm{Y}$ respectively. Since $S=\phi$ there exists a $j_{\text {。 }}$ such that $\mathcal{S}_{j} \cap\left(K_{1} \times K_{2}\right)=\phi$ for all $j \geqq j_{\circ}$. Thus, if $p \in K_{1}$ then $f_{j}(p) \notin K_{2}$ for all $j \geqq j_{\circ}$. Consequently we have that $f_{j}\left(K_{1}\right) \cap K_{2}=\phi$ for all $j \geqq j_{0}$, so that $\left\{f_{j}\right\}$ is compactly divergent.

(2) In the case that $e=\phi$ :

For every point $p \in \mathrm{X}, S(p) \neq \phi$ and it consists of exactly one point. From this, we can define a mapping $\eta$ from $\mathrm{X}$ to $\mathrm{Y}$ such that $S$ is a graph of $\eta$. We shall show that the mapping $\eta$ is continuous in $\mathrm{X}$. Suppose that $\eta$ is not continuous at $p_{\circ}$. Then there exists a sequence $\left\{p_{j}\right\}$ of $\mathrm{X}$ such that $p_{j} \rightarrow p_{\circ}$ and $d_{\mathrm{Y}}\left(\eta\left(p_{j}\right), \eta\left(p_{\circ}\right)\right) \geqq \delta$ for some positive number $\delta$. Since $\left(p_{\circ}, \eta\left(p_{\circ}\right)\right) \in S$, there exists a sequence $\left\{\left(q_{j}, w_{j}\right)\right\}$ such that $\left(q_{j}, w_{j}\right) \in \mathcal{G}_{j}$, $q_{j} \rightarrow p_{\circ}$ and $w_{j} \rightarrow \eta\left(p_{\circ}\right)$. Put $\varepsilon=\frac{1}{5} \delta$, then there exists a $j_{\circ}$ such that 
$d_{\mathrm{Y}}\left(w_{j}, \eta\left(p_{\circ}\right)\right)<\varepsilon$ and $d_{\mathrm{X}}\left(q_{j}, p_{\mathrm{o}}\right)<\varepsilon$ for all $j \geqq j_{\circ}$. Moreover, since $\widetilde{F}$ is equicontinuous at $p_{\circ}$, there exists a positive number $r$ such that $d_{\mathrm{X}}\left(f_{j}(p)\right.$, $\left.f_{j}\left(p_{\circ}\right)\right)<\varepsilon$ for all $p \in B_{\mathbf{X}}\left(p_{\circ}: r\right)$ and for all $j$. Take a point $p^{\prime} \in\left\{p_{j}\right\}$ such that $p^{\prime} \in B_{\mathrm{X}}\left(p_{\circ}: \frac{r}{2}\right)$. Then

$$
d_{\mathrm{Y}}\left(\eta\left(p^{\prime}\right), \eta\left(p_{\circ}\right)\right) \geqq \delta, d_{\mathrm{Y}}\left(f_{j}\left(p^{\prime}\right), f_{j}\left(p_{\circ}\right)\right)<\varepsilon .
$$

Since $\left(p^{\prime}, \eta\left(p^{\prime}\right)\right) \in S$, we can take a sequence $\left\{\left(t_{j}, s_{j}\right)\right\}$ such that $\left(t_{j}, s_{j}\right) \in \mathscr{G}_{j}, t_{j} \rightarrow p^{\prime}$ and $s_{j} \rightarrow \eta\left(p^{\prime}\right)$. Then there exists a $k_{0}$ such that $d_{\mathrm{X}}\left(t_{j}, p^{\prime}\right)$ $<\frac{r}{2}, d_{\mathrm{Y}}\left(s_{j}, \eta\left(p^{\prime}\right)\right)<\varepsilon$ for all $j \geqq k_{\circ}$. Since $t_{j} \in B_{\mathrm{X}}\left(p_{\circ}: r\right)$ for all $j \geqq k_{\circ}$, it holds that

$$
d_{\mathrm{Y}}\left(f_{j}\left(t_{j}\right), f_{j}\left(p_{\circ}\right)\right)<\varepsilon, d_{\mathrm{Y}}\left(f_{j}\left(p_{0}\right), f_{j}\left(q_{j}\right)\right)<\varepsilon
$$

for all $j$ larger than $j_{\circ}$ and $k_{\circ}$. Since $w_{j}=f_{j}\left(q_{j}\right)$, we have

$$
\begin{aligned}
d_{\mathrm{Y}}\left(\eta\left(p^{\prime}\right), \eta\left(p_{\circ}\right)\right) \leqq & d_{\mathrm{Y}}\left(\eta\left(p^{\prime}\right), s_{j}\right)+d_{\mathrm{Y}}\left(s_{j}, f_{j}\left(p_{\circ}\right)\right)+d_{\mathrm{Y}}\left(f_{j}\left(p_{\circ}\right), w_{j}\right) \\
& +d_{\mathrm{Y}}\left(w_{j}, \eta\left(p_{\circ}\right)\right)<4 \varepsilon=\frac{4}{5} \delta
\end{aligned}
$$

for all $j$ larger than $j_{\circ}$ and $k_{\circ}$. This is a contradiction and we have that $\eta$ is continuous in $\mathrm{X}$.

Now let $K_{1}$ be a compact set in $\mathrm{X}$. Take a compact set $K_{2}$ in $\mathrm{Y}$ and put $K=K_{1} \times K_{2}$. Then

for any positive number $\varepsilon$, there exists $a j$ 。 such that

$$
\mathcal{S}_{j} \cap K \subset \bigcup_{p \in K_{1}}\{p\} \times B_{\mathrm{Y}}(\eta(p): \varepsilon)
$$

for all $j \geqq j_{\circ}$.

In fact, suppose that there exists a positive number $\delta$ such that

$$
\left\{j ; \mathbb{B}_{j} \cap K-\bigcup_{p \in K_{1}}\{p\} \times B_{\mathrm{Y}}(\eta(p): \delta) \neq \phi\right\}
$$

is infinite. Then there exists a sequence $\left\{\left(p_{\nu_{j}}, q_{\nu_{j}}\right)\right\}$ such that

$$
\left(p_{\nu_{j}}, q_{\nu_{j}}\right) \in \circlearrowleft \nu_{j} \cap K-\bigcup_{p \in K_{1}}\{p\} \times B_{Y}(\eta(p): \delta)
$$


We may assume that $p_{\nu_{j}} \rightarrow p \in K_{1}, q_{\nu_{j}} \rightarrow q \in K_{2}$. Since $p_{\nu_{j}} \in K_{1}$, we have that $q_{\nu_{j}} \notin B_{\mathrm{Y}}\left(\eta\left(p_{\nu_{j}}\right): \delta\right)$. That is $d_{\mathrm{Y}}\left(q_{\nu_{j}}, \eta\left(p_{\nu_{j}}\right)\right) \geqq \delta$. On the other hand, since $\eta$ is continuous, there exists a positive number $r$ such that

$$
\eta\left(B_{\mathbf{X}}(p: r)\right) \subset B_{\mathbf{Y}}\left(\eta(p): \frac{\delta}{4}\right)
$$

Take a $j_{\text {。 }}$ such that $\left(p_{\nu_{\jmath}}, q_{\nu_{\jmath}}\right) \in B_{\mathrm{X}}(p: r) \times B_{\mathrm{Y}}\left(\eta(p): \frac{\delta}{4}\right)$ for all $j \geqq j_{\circ}$. Then we have

$$
d_{\mathbf{Y}}\left(q_{\nu_{\jmath}}, \eta\left(p_{\nu_{\jmath}}\right)\right) \leqq d_{\mathbf{Y}}\left(q_{\nu_{\jmath}}, \eta(p)\right)+d_{\mathbf{Y}}\left(\eta(p), \eta\left(p_{\nu_{\jmath}}\right)\right) \leqq \frac{\delta}{2}
$$

for all $j \geqq j_{\circ}$. This contradicts the fact that $d_{\mathbf{Y}}\left(q_{\nu_{j}}, \eta\left(p_{\nu_{j}}\right)\right) \geqq \delta$, so that

$$
\text { (S) }_{j} \cap K \subset \bigcup_{p \in K_{1}}\{p\} \times B_{\mathbf{Y}}(\eta(p): \varepsilon)
$$

for all sufficiently large $j$. Put

$$
K_{2}=\overline{\eta\left(K_{1}\right)^{(1)}}
$$

then the following statement holds:

There exists $a j_{\circ}$ such that $\mathbb{B}_{j} \cap\left(K_{1} \times \mathrm{Y}\right)=\mathbb{S}_{j} \cap\left(K_{1} \times K_{2}\right)$ for all $j \geqq j_{\circ}$.

In fact, suppose that this statement does not hold. Then there exists a sequence $\left\{\left(p_{\nu_{j}}, q_{\nu_{j}}\right)\right\}$ such that

$$
\left(p_{\nu_{j}}, q_{\nu_{j}}\right) \in \mathbb{S} \nu_{j} \cap\left(K_{1} \times \mathrm{Y}\right)-\text { \& }_{j} \nu_{j} \cap\left(K_{1} \times K_{2}\right) .
$$

Since $p_{\nu j} \in K_{1}$, this means that $q_{\nu_{j}} \notin K_{2}$, that is $d_{\mathrm{Y}}\left(q_{\nu_{\jmath}}, \eta\left(K_{1}\right)\right)>1$. For simplicity, we may assume that $p_{\nu_{j}} \rightarrow p_{\circ} \in K_{1}$. Since $\left(p_{\circ}, \eta\left(p_{\circ}\right)\right) \in S$ and since $\left\{\& \nu_{j}\right\}$ converges geometrically to $S$, there exists a sequence $\left\{\left(t_{\nu_{j}}, s_{\nu_{j}}\right)\right\}$ such that $\left(t_{\nu_{j}}, s_{\nu_{j}}\right) \in \mathscr{S}_{\nu_{j}}, t_{\nu_{j}} \rightarrow p_{\circ}$ and $s_{\nu_{j}} \rightarrow \eta\left(p_{\circ}\right)$. Take a positive number $r$ such that $d_{\mathrm{Y}}\left(f_{\nu}\left(p^{\prime}\right), f_{\nu j}\left(p_{\circ}\right)\right)<\frac{1}{4}$ for all $p^{\prime} \in B_{\mathrm{X}}\left(p_{\circ}: r\right)$ and for all $j$. Then there exists a $j_{\circ}$ such that $p \nu_{j}, t_{\nu_{j}} \in B_{\mathbf{X}}\left(p_{\circ}: r\right)$ and $d_{\mathrm{Y}}\left(s_{\nu}, \eta\left(p_{\circ}\right)\right)<\frac{1}{4}$ for all $j \geqq j_{\circ}$. It holds that 


$$
\begin{aligned}
d_{Y}\left(q_{\nu,}, \eta\left(p_{\circ}\right)\right) \leqq & d_{Y}\left(q_{\nu_{j}}, f_{\nu_{j}}\left(p_{\circ}\right)\right)+d_{\mathrm{Y}}\left(f_{\nu_{j}}\left(p_{\circ}\right), s_{\nu \jmath}\right) \\
& +d_{Y}\left(s_{\nu,}, \eta\left(p_{\circ}\right)\right)<\frac{3}{4}
\end{aligned}
$$

for all $j \geqq j_{\circ}$. This implies that $d_{Y}\left(q_{\nu_{j}}, \eta\left(K_{1}\right)\right)<\frac{3}{4}$ and this is a contradiction. Summarizing these facts, we have that for any compact set $K_{1} \subset \mathrm{X}$ and for any positive number $\varepsilon$ there exists a $j$ 。 such that

$$
\mathbb{B S}_{j} \cap\left(K_{1} \times Y\right)=\mathbb{S}_{j} \cap\left(K_{1} \times K_{2}\right) \cap \bigcup_{p \in K_{1}}\{p\} \times B_{Y}(\eta(p): \varepsilon)
$$

for all $j \geqq j_{0}$. Thus $\left\{f_{j}\right\}$ converges uniformly to $\eta$ on $K_{1}$. This complete the proof of Theorem 1 .

2. In this section we consider the case of holomorphic functions. As a consequence of Theorem 1 , we have

Corollary of Theorem $\mathbb{1}$. Let $\mathrm{D}$ be a domain in $\mathbb{C}^{n}$ and let $\mathfrak{F}$ be a family of holomorphic functions in D. If $\mathfrak{F}$ is equicontinuous at each point of $\mathrm{D}$, then $\mathfrak{F}$ is normal in $\mathrm{D}$.

Now let $\mathrm{D}$ be a domain in $\mathbb{C}^{n}$.

Definition 5. Let $\left\{A_{\nu}\right\}$ be a sequence of principal analytic sets in D. This sequence is called to converge analytically to an analytic set $A$ if and only if given a point $p \in \mathrm{D}$, there exists a neighbourhood $U$ of $p$ and holomorphic functions $\left\{f_{\nu}\right\}, f$ in $U$ such that:

(i) $f$ is not identically zero.

(ii) $A_{\nu} \cap U=\left\{q \in U ; f_{\nu}(q)=0\right\}, A \cap U=\{q \in U ; f(q)=0\}$.

(iii) The sequence $\left\{f_{\nu}\right\}$ converges uniformly to $f$.

From this definition, the following remarks are easily seen.

(1) If $A$ is not empty, then $A$ is a principal analytic set in $\mathrm{D}$.

(2) If a sequence $\left\{p_{\nu}\right\}$ with $p_{\nu} \in A_{\nu}$ has accumulating points, then they belong to $A$.

(3) The sequence $\left\{A_{\nu}\right\}$ converges geometrically to $A$.

Definition 6. A family $\mathfrak{F}$ of holomorphic functions in $D$ is called to be bounded at $p \in \mathrm{D}$ if and only if the set $\mathfrak{F}(p)=\{f(p) ; f \in \mathfrak{F}\}$ is bounded 
in the complex plane.

Definition 7. An analytic set $A$ in $\mathrm{D} \times C(w)$ is called to be fine in $w$ at $p$ if and only if the set $A(p)=\left\{(p, w) \in \mathbb{C}^{n+1}\right\} \cap A$ has no finite accumulating point, where $C(w)$ is a complex plane with coordinate $w$.

From this definition, as is easily seen, $A$ is not fine in $w$ at $p$ if and only if $A$ includes the complex plane $\{(z, w) ; z=p\}$. For simplicity, we consider the case of two complex variables $x, y$.

Lemma 3. Let $\left\{f_{j}\right\}$ be a sequence of holomorphic functions in D satisfying the following conditions:

(i) $\left\{f_{j}\right\}$ is bounded at each point of $\mathrm{D}$.

(ii) The sequence $\left\{\mathscr{S}_{j}\right\}$ of graphs of $\left\{f_{j}\right\}$ converges analytically to an analytic set $A$ in $\mathrm{D} \times \mathbb{C}(w)$.

Then $\left\{f_{j}\right\}$ converges uniformly to a holomorphic function on every compact set in $\mathrm{D}$.

Proof ${ }^{3)}$. Let $E=\{p \in \mathrm{D} ; A$ is not fine in $w$ at $p\}$. Then

$E$ is a proper analytic set in $\mathrm{D}$.

In fact, since $A$ is a proper analytic set in $\mathrm{D} \times \mathbb{C}(w), E \subsetneq \mathbb{C}$. Take a point $p \in \mathrm{D}$ and a polydisc $\Delta \subset \mathrm{D}$ with center at $p$. Since $\Delta \times \mathbb{C}$ is a Cousin II domain, there exists a holomorphic function $\varphi(x, y, w)$ in $\Delta \times \mathbb{C}$ such that

$$
A \cap(\Delta \times \boldsymbol{C})=\{(x, y, w) \in \Delta \times \mathbb{C} ; \varphi(x, y, w)=0\}
$$

Then it is easily seen that

$$
\begin{aligned}
E \cap \Delta & =\{(x, y) \in \Delta ; \varphi(x, y, c)=0 \text { for all complex numbers } c\} \\
& =\bigcap_{c \in C}\{(x, y) \in \Delta ; \varphi(x, y, c)=0\} .
\end{aligned}
$$

Thus $E$ is a proper analytic set in D.

Now, we show that the sequence $\left\{f_{j}\right\}$ is uniformly bounded on every

3) This argument was suggested by Prof. H. Fujimoto as an alternative to a more complicated original one of the author. We wish to thank him for his kind advice. 
compact set in D. To prove this, we have only to show that for any point $p \in \mathrm{D}$ there exists a neighbourhood $U$ of $p$ such that $\left\{f_{j}\right\}$ is uniformly bounded on $U$. Let $p_{\circ} \in \mathrm{D}-E$. Since $\left\{f_{j}\left(p_{\circ}\right)\right\}$ is bounded, there exists a subsequence of $\left\{f_{j}\left(p_{0}\right)\right\}$ which converges to a complex number $q_{0}$. Since $\left(p_{\circ}, q_{\circ}\right) \in A$, there exists a neighbourhood $U=U_{1} \times U_{2}$ of $\left(p_{\circ}, q_{\circ}\right)$ with $U_{1} \subset \mathrm{D}-E$ and holomorphic functions $\left\{\psi_{j}\right\}, \psi$ in $U$ such that

$$
\begin{gathered}
\mathcal{S}_{j} \cap U=\left\{(x, y, w) \in U ; \psi_{j}(x, y, w)=0\right\} \\
A \cap U=\{(x, y, w) \in U ; \psi(x, y, w)=0\}
\end{gathered}
$$

and that the sequence $\left\{\psi_{j}\right\}$ converges uniformly to $\psi$ on $U$. Since $p_{\circ} \notin E$, $\psi\left(p_{\circ}, w\right)$ is not identically zero. Therefore there exists a small positive number $r$ such that $\psi\left(p_{\circ}, w\right) \neq 0$ if $\left|w-q_{\circ}\right|=r$. Thus if we choose $U_{1}$ sufficiently small, we have $\psi \neq 0$ on $\bar{U}_{1} \times \Theta$, where $\Theta=\{w \in C ; \mid w-q$ 。 $=r\}$. Put $m=\min \left\{|\psi(p, w)| ;(p, w) \in \bar{U}_{1} \times \Theta\right\}$. Since $\left\{\psi_{j}\right\}$ converges

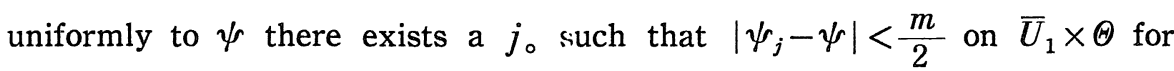
all $j \geqq j_{\circ}$. Then for any point $p \in U_{1}$, by the theorem of Hurwitz, the equation $\psi_{j}(p, w)=0$ has at least one root $w_{j}$ in the disc $\left\{w \in C ;\left|w-q_{\circ}\right|\right.$ $<r\}$ for all $j \geqq j_{\circ}$. Thus $\left\{f_{j}\right\}$ is uniformly bounded on $U_{1}$. Let $p_{\circ} \in E$. Since $E$ is a proper analytic set, by a linear change of coordinate if necessary, we can choose a polydisc $U=U_{1} \times U_{2}$ of $p_{\text {。 }}$ such that $\left(\partial U_{1} \times \partial U_{2}\right) \cap E$ $=\phi$. Then $\left\{f_{j}\right\}$ is uniformly bounded on $\partial U_{1} \times \partial U_{2}$ and by the maximal principle $\left\{f_{j}\right\}$ is uniformly bounded on $U_{1} \times U_{2}$.

Now by the theorem of Montel, any sequence of $\left\{f_{j}\right\}$ has a convergent subsequence. To prove that $\left\{f_{j}\right\}$ converges uniformly on every compact set in $\mathrm{D}$, we have only to show that the limit function is independ of the choice of the convergent sequence. Let $\left\{f_{j}^{(1)}\right\}$ and $\left\{f_{j}^{(2)}\right\}$ be two sequences of $\left\{f_{j}\right\}$ which converge to $f^{(1)}$ and $f^{(2)}$ respectively on every compact set in D. Then it is easily seen that $A=$ graph of $f^{(1)}=$ graph of $f^{(2)}$, so that $f^{(1)}=f^{(2)}$. This complete the proof of Lemma 3 .

Definition 8. A family of principal analytic sets in $D$ is called to be analytically normal in $\mathrm{D}$ if and only if given a sequence of the family, there exists a subsequence which converges analytically to an analytic set in $\mathrm{D}$. 
Definition 9. Let $\mathfrak{S}$ (resp. $\mathfrak{F}$ ) be a family of principal analytic sets (resp. holomorphic functions) in D. Then $\mathrm{D}$ is called a domain of normality of $\mathfrak{S}$ (resp. $\mathfrak{F}$ ) if and only if $\mathfrak{S}$ (resp. $\mathfrak{F}$ ) is normal in $\mathrm{D}$ and for any schlicht domain $\tilde{\mathrm{D}}$ in $\boldsymbol{C}^{2}$ such that $\mathrm{D} \subsetneq \tilde{\mathrm{D}}, \mathfrak{S}$ (resp. $\mathfrak{F}$ ) is no longer normal in $\tilde{\mathrm{D}}$.

For a domain of normality of a family of holomorphic functions, the so-called conjecture of G. Julia is well known ${ }^{4}$. In connection with this conjecture, we have the following

Theorem 2. Let $\mathfrak{F}$ be a family of holomorphic functions in D. Suppose that $\mathfrak{F}(p)$ is bounded at each point $p$ of $\mathrm{D}$. If $\mathrm{D} \times \boldsymbol{C}$ is a domain of normality of a family $\mathbb{S}_{\mathfrak{F}}$ of graphs of $\mathfrak{F}$, then $\mathrm{D}$ is a domain of holomorphy.

Proof. Suppose that D is not a domain of holomorphy. Then since D is not holomorphycally convex, there exists a compact set $K$ in D such that

$$
\begin{aligned}
& \tilde{K}=\left\{(x, y) \in \mathrm{D} ;|f(x, y)| \leqq \sup _{K}|f|\right. \\
& \text { for all holomorphic functions in } \mathrm{D}\}
\end{aligned}
$$

is not compact in D. Put $\rho=d(K, \partial \mathrm{D})$ and take a point $p_{\circ}=\left(x_{\circ}, y_{\circ}\right) \in \tilde{K}$ such that $d\left(p_{\circ}, \partial \mathrm{D}\right)<\frac{\rho}{2}$. It is well known that any holomorphic function in $\mathrm{D}$ is also holomorphic in the polydisc $\Delta=\left\{(x, y) \in \mathbb{C}^{2} ;\left|x-x_{\mathrm{o}}\right|<\rho\right.$, $\left.\left|y-y_{\circ}\right|<\rho\right\} . \quad$ Put $\Delta^{\prime}=\left\{(x, y) \in \mathbb{C}^{2} ;\left|x-x_{\circ}\right|<\frac{2}{3} \rho,\left|y-y_{\circ}\right|<\frac{2}{3} \rho\right\}$. Since $d\left(p_{\circ}, \partial \mathrm{D}\right)<\frac{\delta}{2}, \Delta^{\prime}-\mathrm{D} \neq \phi$. We shall show that

$\mathbb{S}_{\Re}$ is normal in $\left(\mathrm{D} \cup \Delta^{\prime}\right) \times \mathbb{C}$.

In fact, take a sequence $\left\{\mathscr{S}_{\nu}\right\}$ from $\mathscr{S}_{\overparen{F}}$. Since $\mathscr{E}_{\mathfrak{F}}$ is normal in $\mathrm{D} \times \boldsymbol{C}$, there exists a subsequence $\left\{\mathscr{S}_{\nu_{j}}\right\}$ which converges analytically to an analytic set $\mathrm{A}$ in $\mathrm{D} \times \boldsymbol{C}$. Let $f_{\nu j}$ be the function which represents the graph $\mathbb{S B}_{\nu j}$. Then by Lemma $3,\left\{f_{\nu_{j}}\right\}$ converges uniformly to a holomorphic function $\eta$ on every compact set in D. Expand $f_{\nu_{j}}$ into the convergent Taylor series in $\Delta^{\prime}$ :

4) See, [5] page 38 . 


$$
f_{\nu_{j}}(x, y)=\Sigma a_{k_{1}, k_{2}}^{\left(\nu_{j}\right)}\left(x-x_{\circ}\right)^{k_{1}}\left(y-y_{\circ}\right)^{k_{2}},
$$

where

$$
a_{k_{1}, k_{2}}^{(\nu, j)}=\frac{1}{k_{1} ! k_{2} !} \frac{\partial^{k_{1}+k_{2}} f_{\nu_{j}}\left(p_{\circ}\right)}{\partial x^{k_{1}} \partial y^{k_{2}}}
$$

Put $K^{\prime}=\overline{K^{(r)}}$, where $r=\frac{3}{4} \rho$. Since $K^{\prime}$ is compact, it holds that $\sup _{\nu j} \sup _{K^{\prime}}$ $\left|f_{\nu j}\right|=M$. Then by the estimation of Cauchy, we have

$$
\left|a_{k_{1}, k_{2}}^{(\nu, j)}\right| \leqq \frac{1}{k_{1} ! k_{2} !} \sup _{p \in K}\left|\frac{\partial^{k_{1}+k_{2}} f \nu_{j}(p)}{\partial x^{k_{1}} \partial y^{k_{2}}}\right| \leqq \frac{M}{\left(\frac{3}{4} \rho\right)^{k_{1}+k_{2}}}
$$

Therefore $\left\{f_{\nu_{j}}\right\}$ is uniformly bounded on $\Delta^{\prime}$. Thus there exists a subsequence of $\left\{f_{\nu_{j}}\right\}$ which converges uniformly to a holomorphic function in $\Delta^{\prime}$. For simplicity, we may assume that $\left\{f_{\nu j}\right\}$ converges uniformly to $\tilde{\eta}$ in $\Delta^{\prime}$. Since $\tilde{\eta}=\eta$ in $\Delta^{\prime} \cap \mathrm{D}$, the holomorphic function $\xi$ such that $\xi=\eta$ in $\mathrm{D}$ and $\xi=\tilde{\eta}$ in $\Delta^{\prime}$ is determined. Then $\left\{\mathscr{S}_{\nu j}\right\}$ converges analytically to the analytic set $B=\left\{(p, \xi(p)) ; p \in \Delta^{\prime} \cup D\right\}$ in $\left(\Delta^{\prime} \cup D\right) \times C$. Since $D \times C$ is a domain of holomorphy of $\mathbb{S}_{\mathfrak{F}}$, this is a contradiction.

Remark of Lemma 3. In Lemma 3, we omit the condition of the boundedness of $\left\{f_{j}\right\}$ at each point and consider under the condition only of an analytic convergence of graphs. Put

$$
\Omega=\left\{p \in \mathrm{D}-E ;\left\{f_{j}(p)\right\} \text { is bounded }\right\}
$$

It is easily seen that the boundedness at $p$ is equivalent to the condition that $A(p) \neq \phi$. Moreover, since $A$ is fine in $w$ at $p \in \mathrm{D}-E$ it is easy to see that $\Omega=\pi(A)-E$, where $\pi$ is the projection given by $\pi(p, w)=p$. Therefore $\Omega$ is open. Thus if $\Omega$ is not empty, there exists a holomorphic function $\eta$ in $\Omega$ such that $\left\{f_{j}\right\}$ converges uniformly to $\eta$ on every compact set in $\Omega$. If $\Omega=\phi$, then $[(\mathrm{D}-E) \times C] \cap A=\phi$. Since $\left\{\mathscr{S}_{j}\right\}$ converges analytically to $A$, for any compact set $K_{1}$ in $\mathrm{D}-E$ and for any positive number $a$, there exists a $j_{\circ}$ such that $\mathscr{S}_{j} \cap K=\phi$ for all $j \geqq j_{\circ}$, where $K$ $=K_{1} \times\{w \in C ;|w| \leqq a\}$. Thus if $p \in K_{1}$ then $\left(p, f_{j}(p)\right) \notin K$, i.e., $\left|f_{j}(p)\right|$ $>a$ for all $j \geqq j_{\circ}$. This means that $\left\{f_{j}\right\}$ is compactly divergent in $\mathrm{D}-\boldsymbol{E}$. 
Let $\mathrm{D}$ be a domain of holomorphy and let $\Omega \neq \phi$, then it is easily seen that $\Omega$ is a holomorphic open set, i.e., any connected component of $\Omega$ is a domain of holomorphy.

3. In this section, we consider an application of the theorem of G. Julia:

Theorem ([6], page 67). Let $\mathrm{D}$ be a domain in $\boldsymbol{C}^{2}$ and let $\mathfrak{F}$ be $a$ family of holomorphic functions in $\mathrm{D}$. If every function $f \in \mathfrak{F}$ does not take two fixed different values, then $\mathfrak{F}$ is normal in $\mathrm{D}$.

Lemma 4. Let $\mathrm{D}$ be a domain in $\boldsymbol{C}^{2}$ and let $\left\{f_{\nu}\right\}, f$ be holomorphic functions in D. Let $\Lambda=\{a, b, c\}$ be a set consisting of three different complex numbers. Suppose that for any $\alpha \in \Lambda$, the sequence $\left\{A_{\nu, \alpha}\right\}$ of analytic sets in D given by

$$
A_{\nu, \alpha}=\left\{(x, y) \in \mathrm{D} ; f_{\nu}(x, y)=\alpha\right\},
$$

converges geometrically to an analytic set $A_{\alpha}=\{(x, y) \in \mathrm{D} ; f(x, y)=\alpha\}$. Then $\left\{f_{\nu}\right\}$ is normal in $\mathrm{D}$.

Proof. Take a point $p \in \mathrm{D}$. If $f(p) \notin \Lambda$, then there exists a connected open neighbourhood $V$ of $p$ such that $V \ll \subset$ D and $f(\bar{V}) \cap \Lambda=\phi$. Therefore, $\Lambda-f(\bar{V})=\Lambda$. If $f(p) \in \Lambda$, we can choose a connected open neighbourhood $U$ of $p$ such that $U \ll \mathrm{D}$ and $f(\bar{U}) \cap[\Lambda-\{f(p)\}]=\phi$. In any case we can choose a connected open neighbourhood $U$ of $p$ such that $U \ll \mathrm{D}$ and that $\Lambda-f(\bar{U})$ contains at least two complex numbers. Let $\{a, b\} \subset A-f(\bar{U})$, then $A_{a} \cap \bar{U}=\phi$ and $A_{b} \cap \bar{U}=\phi$. Since $\left\{A_{\nu, a}\right\},\left\{A_{\nu, b}\right\}$ converge geometrically to $A_{a}, A_{b}$ respectively, there exists $a \nu_{0}$ such that $A_{\nu, a} \cap \bar{U}=\phi, A_{\nu, b} \cap \bar{U}$ $=\phi$ for all $\nu \geqq \nu_{0}$. That is, the sequence $\left\{f_{\nu}\right\}_{\nu \geqq \nu_{0}}$ does not take two different values $a, b$ in $U$. Thus by the theorem of Julia, it is normal in $U$. Since $p$ is an arbitrary point of $\mathrm{D},\left\{f_{\nu}\right\}$ is normal at each point of $\mathrm{D}$. Then by the diagonal method $\left\{f_{\nu}\right\}$ is normal in $\mathrm{D}$.

Definition 10. A set $\omega$ is called a set of uniqueness if and only if any holomorphic function in $\mathrm{D}$ which is zero on $\omega$ is identically zero.

Theorem 3. Let $\left\{f_{\nu}\right\}, f$ be holomorphic functions in $\mathrm{D}$ and let $f$ 
be not constant. Let $\omega$ be a set of uniqueness. Suppose that for any $a \in f(\omega)$ the sequence $\left\{A_{\nu, a}\right\}$ of analytic sets in $\mathrm{D}$ converges geometrically to an analytic set $A_{a}$, then $\left\{f_{\nu}\right\}$ converges uniformly to $f$ on every compact set in $\mathrm{D}$, where $A_{\nu, a}, A_{a}$ are the same as that in Lemma 4.

Proof. First, the set $f(\omega)$ contains infinitely many complex numbers. In fact, suppose that $f(\omega)$ contains only finitely many complex numbers. Let $f(\omega)=\left\{a_{1}, a_{2}, \ldots, a_{m}\right\}$. Put $S_{i}=\left\{p \in \mathrm{D} ; f(p)=a_{i}\right\}$. Then the analytic set $S=\cup S_{i}$ is given by

$$
S=\left\{p \in \mathrm{D} ; g(p)=\prod_{i=1}^{i=m}\left[f(p)-a_{i}\right]=0\right\}
$$

Since $f$ is not constant $g$ is not also constant. But since $S \supset \omega$ and since $g=0$ on $S, g$ is identically zero, this is a contradiction. Thus by Lemma $4\left\{f_{\nu}\right\}$ is normal in $\mathrm{D}$. To prove that the sequence $\left\{f_{\nu}\right\}$ converges uniformly to $f$ on every compact set in $\mathrm{D}$, we have only to show that any sequence of $\left\{f_{\nu}\right\}$ contains a subsequence which converges uniformly to $f$ on every compact set in D. Take a sequence $\left\{f_{\nu j}\right\}$ of $\left\{f_{\nu}\right\}$. We may assume that $\left\{f_{\nu j}\right\}$ is compact uniform convergence or compact divergence. Since $\left\{A_{\nu, a}\right\}$ converges geometrically to $A_{a}$ and since $A_{a}$ is not empty, the case of compact divergence does not occur. Let $\left\{f_{\nu j}\right\}$ converge uniformly to a holomorphic function $h$ on every compact set in D. Let $p_{\circ} \in \omega$ and let $a=f\left(p_{\circ}\right)$, then since $p_{\circ} \in A_{a}$ there exists a sequence $\left\{p_{\nu_{j}}\right\}$ such that $p_{\nu_{j}}$ $\in A_{\nu_{j}, a}$ and $p_{\nu_{j}} \rightarrow p_{\circ}$. Let $K=\left\{p_{\circ}, p_{\nu_{j}} ; j=1,2, \ldots\right\}$. Since $K$ is compact, for any positive number $\varepsilon$ there exists a $j$ 。 such that

$$
\sup _{p \in K}\left\{\left|f_{\nu_{j}}(p)-h(p)\right|\right\}<\varepsilon,\left|h\left(p_{\nu_{j}}\right)-h\left(p_{\circ}\right)\right|<\varepsilon
$$

for all $j \geqq j_{\circ}$. Then we have

$$
\left|f_{\nu_{j}}\left(p_{\nu_{j}}\right)-h\left(p_{\circ}\right)\right| \leqq\left|f_{\nu_{j}}\left(p_{\nu_{j}}\right)-h\left(p_{\nu_{j}}\right)\right|+\left|h\left(p_{\nu_{j}}\right)-h\left(p_{\circ}\right)\right|<2 \varepsilon .
$$

Since $p_{\nu_{j}} \in A_{\nu_{j}, a}$ and since $f_{\nu_{j}}\left(p_{\nu_{j}}\right)=a=f\left(p_{\circ}\right)$, we have $f\left(p_{\circ}\right)=h\left(p_{\circ}\right)$. That is, $f=h$ on $\omega$, so that $f=h$ on $\mathrm{D}$.

Remark. In Theorem 3, we can not omit the condition that $f$ is not constant. But since Lemma 4 holds in the case that $f$ is constant, by the 
same method we can prove the following:

Let $U$ be an open set in the complex plane such that $U \cap f(\mathrm{D}) \neq \phi . \quad$ If $\left\{A_{\nu, a}\right\}$ converges geometrically to $A_{a}$ for all $a \in U$, then $\left\{f_{\nu}\right\}$ converges uniformly to $f$ on every compact set in D.

Now we consider the case of one complex variable. If a sequence $\left\{f_{j}\right\}$ of holomorphic functions in a domain $\mathrm{D} \subset \boldsymbol{C}$ converges to a non constant holomorphic function $f$ on every compact set in $\mathrm{D}$, then for any point $p \in \mathrm{D}$ and for any complex number $a$, there exists a positive number $r_{\circ}$ which satisfies the following property:

For any $r$ with $0<r<r_{0}$, there exists $a j_{\circ}$ such that the number of $a$ points of $f_{j}$ in the disc $\{z \in C ;|z-p|<r\}$ are equal for all $j \geqq j_{\circ}$, counted according to multiplicities.

In fact, if $f(p) \neq a$, then there exists a neighbourhood $U$ of $p$ such that $U \ll \mathrm{D}$ and $\min \{|f(z)-a| ; z \in \bar{U}\}=\delta>0$. Since $\left\{f_{j}\right\}$ converges uniformly to $f$ on $\bar{U}$ there exists a $j$ 。 such that

$$
\max \left\{\left|f_{j}(z)-f(z)\right| ; z \in \bar{U}\right\}<\frac{\delta}{2}
$$

for all $j \geqq j_{0}$. Then

$$
\left|f_{j}(z)-a\right| \geqq|f(z)-a|-\left|f_{j}(z)-f(z)\right|>\frac{\delta}{2}
$$

for all $j \geqq j_{\circ}$ and $z \in \bar{U}$. That is $f_{j}(z) \neq a$ in $\bar{U}$ for all $j \geqq j_{\circ}$. If $f(p)=a$, then this is just the theorem of Hurwitz.

Since Theorem 3 holds for a domain in the complex plane, as the converse of the above, we have

Corollary of Theorem 3. Let $\left\{f_{j}\right\}, f$ be holomorphic functions in a domain $\mathrm{D}$ in $\boldsymbol{C}$ and let $f$ be not constant. Suppose that for any point $p \in \mathrm{D}$ and for any complex number a, there exists a positive number $r_{\circ}$ satisfying the following property:

For any $r$ with $0<r<r_{0}$, there exists $a j_{\circ}$ such that the number of $a$ - 
points of $f_{j}$ and $f$ are equal in the disc $\{z \in C ;|z-p|<r\}$ for all $j \geqq j_{0}$, counted according to multiplicities.

Then $\left\{f_{j}\right\}$ converges uniformly to $f$ on every compact set in D.

Proof. We have only to show that for every point $p \in \mathrm{D}$, the sequence $\left\{A_{j}\right\}$ of analytic sets given by $A_{j}=\left\{z \in \mathrm{D} ; f_{j}(z)=f(p)\right\}$ converges geometrically to an analytic set $A=\{z \in \mathrm{D} ; f(z)=f(p)\}$. The condition (i) of geometric convergence is trivial. Let $K$ be a compact set in $\mathrm{D}$ such that $A \cap K=\phi$. For any point $q \in K$, there exists a positive number $\delta$ such that $f(z)-f(p) \neq 0$ in the disc $\Delta=\{z \in \mathrm{D} ;|z-q|<\delta\}$. Evidently we may assume that $\delta<r_{0}$. Then there exists a $j_{\circ}(q)$ such that $f_{j}(z)-f(p) \neq 0$ in the disc $\Delta$ for all $j \geqq j_{\circ}(q)$. Since $K$ is compact we can choose finitely many such discs $\Delta_{\nu}$ with center at $q_{\nu}$ and radius $\delta_{\nu}$ such that $K \subset \cup \Delta_{\nu}$. Put $j_{\circ}=\max j_{\circ}\left(q_{\nu}\right)$. Then since $f_{j}(z)-f(p) \neq 0$ in $K$ for all $j \geqq j_{\circ}$, we have that $A_{j} \cap K=\phi$ for all $j \geqq j_{\circ}$. Thus the condition (ii) is proved.

\section{References}

[1] Alexandroff, P. and Hopf, H., Topologie, Springer, Berlin, 1936.

[2] Gunning R.C. and Rossi, H., Analytic functions of several complex variables, Prentice Hall, 1965.

[3] Hervé, M., Several complex variables, Local theory, Oxford Univ. Press, 1963.

[4] Hirschhowitz, A., Sur l'approximations des hypersurfaces, Annali della Scuola Norm., Pisa, 25, 1971.

[5] Hitotumatu, S., Theory of analytic functions of several complex variables (Japanese), Baihūkan, 1960.

[6] Julia, G., Sur les familles de fonctions analytiques de plusieurs variables, Acta, Math., 47, 1926.

[7] Stolzenberg, G., Volumes, limits, and extensions of analytic varieties, Lecture Note in Math., No. 19, Springer Verlag, 1966. 